\title{
Trends in the study on medical education over the last 10 years, based on paper titles
}

\author{
Seong Yong Kim \\ Department of Medical Education, Yeungnam University College of Medicine, Daegu, Korea
}

Received: April 25, 2019

Revised: May 7, 2019

Accepted: May 8, 2019

Corresponding author:

Seong Yong Kim

Department of Medical Education,

Yeungnam University College of

Medicine, 170, Hyeonchung-ro,

Nam-gu, Daegu 42415, Korea

Tel: +82-53-640-6987

Fax: +82-53-628-4383

E-mail: seongyong@ynu.ac.kr
Medical education research subjects are incredibly diverse and have changed over time. This work in particular aims to compare and analyze research trends in medical education through the words used in the titles of these research papers. Academic Medicine (the journal of the Association of American Medical Colleges), Medical Teacher (the journal of the Association of Medical Education in Europe), the Korean Journal of Medical Education (KIME), and Korean Medical Education Review (KMER) were selected and analyzed for the purposes of this research. From 2009 to 2018, Academic Medicine and Medical Teacher published approximately 10 to 20 times more papers than the KJME and KMER. Frequently used words in these titles include "medical," "student," "education," and "learning." The words "clinical" and "learning" were used relatively often (7.80\% to 13.66\%) in Korean journals and Medical Teacher, but Academic Medicine used these phrases relatively less often ( $6.47 \%$ and $4.41 \%$, respectively). Concern with such various topics as problem-based learning, team-based learning, program evaluations, burnout, e-learning, and digital indicates that Medical Teacher seems to primarily deal with teaching and learning methodologies, and Academic Medicine handles all aspects of medical education. The KJME and KMER did not cover all subjects, as they publish smaller papers. However, it is anticipated that research on new subjects, such as artificial intelligence in medical education, will occur in the near future.

Keywords: Learning; Medical education; Program evaluation; Teaching

\section{Introduction}

Research subjects in medical education are incredibly diverse and have changed with the times. In the past 10 years, the annual conferences held by the Korean Society of Medical Education (KSME) had the following themes: In 2009 (the 25th Annual Conference), the main theme was "From Scholarly Medicine to Professional Competency"; "Medical Humanities" in 2010; "Medical Education: Reflection and Reform" in 2011; "Outcome-Based Medical Education" in 2012; "Challenges for Future Medical Education" in 2013; "Culture and Environment: Medical Education in Korea" in 2014; "Professoring" in 2015; "Students in Medical Education" in 2016; "Future Medical Education and Artificial Intelligence" in 2017; and "A New Horizon for the Doctor Training System in Medical Education" in 2018. Each year, the KSME has decided upon a main theme to match the changes in the medical education and social environments [1].

Many medical education associations exist worldwide, such as the Association of American Medical Colleges (AAMC), the Association of Medical Education in Europe (AMEE), the Association of Medical Schools in Europe, the Asian Medical Education Association, the General Medical Council in the United Kingdom, the Canadian Association for Medical Education, and the Sociedad Española de Educación Médica [2]. These organizations also publish medical education journals, such as Academic Medicine, the journal of the AAMC; BMC Medical Education; 
British Medical Journal; Educacion Medica (in Spanish); the International Journal of Medical Education; JMIR Medical Education; the Journal of Graduate Medical Education; the Journal of Interprofessional Care; MedEdPublish, the AMEE's open-access journal; Medical Education, the ASME's journal; Medical Teacher, another AMEE journal; Teaching and Learning in Medicine; and Research and Development in Medical Education.

In Korea, the KSME publishes the Korean Journal of Medical Education (KJME). Another medical education journal in Korea is the Korean Medical Education Review (KMER), published by the Department of Medical Education at Yonsei University.

This study aimed to compare and analyze trends in medical education research using the words from research paper titles.

\section{Medical education journals}

For the purpose of this paper, two of the world's most prestigious journals - and especially in the United States and Europe were chosen over global studies for an analysis: Academic Medicine, the journal of the AAMC, and Medical Teacher, the journal of the AMEE. The KJME and KMER, two Korean medical education journals, were chosen to examine studies in Korea.

From 2009 to 2018 (Table 1), the KJME published 410 papers, or an average of 41 papers per year, ranging from 30 to 50 per year [3]. The KMER published 205 papers, or an average of 20.5 papers a year, ranging from 9 to 30 per year [4]. Academic Medicine published 4,608 papers, or approximately 460 papers published annually, ranging from 376 to 563 per year [5]. Medical Teacher published 3,745 papers and an average of 374.5 papers a year, ranging from 325 to 442 per year [6]. Academic Medicine and Medical Teacher publish nearly 10 to 20 times more papers than those published in Korean journals.

\section{Frequencies of words used in the titles}

A word cloud (RStudio; RStudio, Boston, MA, USA) uses largesized words to represent words' increasing frequency [7]. When examining the frequencies of words in titles, the larger words in the word cloud primarily involve such connecting words as "and," "of," "for," “to," "in," "the," and "on,” among others (data not shown); therefore, these words occur frequently. After removing these connections, the words "medical," "student," "health," and "education" appeared as larger words. High-frequency words in titles include "medical," "student," "education," and "learning" in the KJME; "medical," "educational," "study," and "development" in the KMER; "medical," "education," "health," and "medicine" in Academic Medicine; and "medical," "education," "student," and "learning" in Medical Teacher (Fig. 1).

The word "medical" appeared in 248 of 410 papers (60.49\%) in the KJME, 117 of 205 papers (57.07\%) in the KMER, 1,405 of 4,608 (30.49\%) papers in Academic Medicine, and 1,602 of 3,745 papers $(42.78 \%)$ in Medical Teacher. The word "education" appeared $21.46 \%, 41.46 \%, 17.17 \%$, and $21.66 \%$ of the time in the KJME, KMER, Academic Medicine, and Medical Teacher, respectively. The word "student" occurred $38.29 \%$ of the time in the KJME, $21.95 \%$ in the KMER, $11.00 \%$ in Academic Medicine, and $25.58 \%$ in Medical Teacher. These words appeared in the titles of the medical education journals because medical schools direct students' medical education (Tables 2, 3).

Most professors and staff in medical schools want to know how to teach students well, how students can learn well, the best way to administer a clinical education, how to improve students' clinical performance, what we must teach students to improve their clinical skill, how to rate students to check their level of education, and how to make beneficial programs for the students, among others. Therefore, the frequency of use for such words as "teaching," "learning," "clinical," "evaluation," "skill," "curriculum," and "program" were investigated (Tables 2, 3). The frequencies in the KJME were as follows: "learning" (13.66\%), "clinical" (13.66\%), "skill” (8.05\%), "program” (7.07\%), "evaluation" (5.85\%), "teaching" (5.61\%), and "curriculum" (3.41\%). The frequencies of use in the KMER were "learning" (12.20\%), "clinical" (7.80\%), "skill" (2.44\%), "program" (9.76\%), "evaluation" (1.95\%), "teaching" (7.80\%), and "curriculum" (9.27\%). The frequencies in Academic Medicine were "learning" (4.41\%), "clinical” (6.47\%), “skill” (2.04\%), “program” (6.32\%),

Table 1. Number of papers in each journal of medical education during last 10 years

\begin{tabular}{lrrrrrrrrrrrr}
\hline & 2009 & 2010 & 2011 & 2012 & 2013 & 2014 & 2015 & 2016 & 2017 & 2018 & Total \\
\hline KJME & 50 & 42 & 41 & 43 & 41 & 40 & 41 & 45 & 30 & 37 & 410 \\
KMER & 9 & 12 & 13 & 16 & 25 & 23 & 23 & 27 & 30 & 27 & 205 \\
Academic Medicine & 419 & 559 & 391 & 376 & 453 & 442 & 417 & 455 & 533 & 563 & 4,608 \\
Medical Teacher & 325 & 327 & 334 & 442 & 383 & 386 & 396 & 340 & 388 & 424 & 3,745 \\
\hline
\end{tabular}

KJME, Korean Journal of Medical Education; KMER, Korean Medical Education Review. 

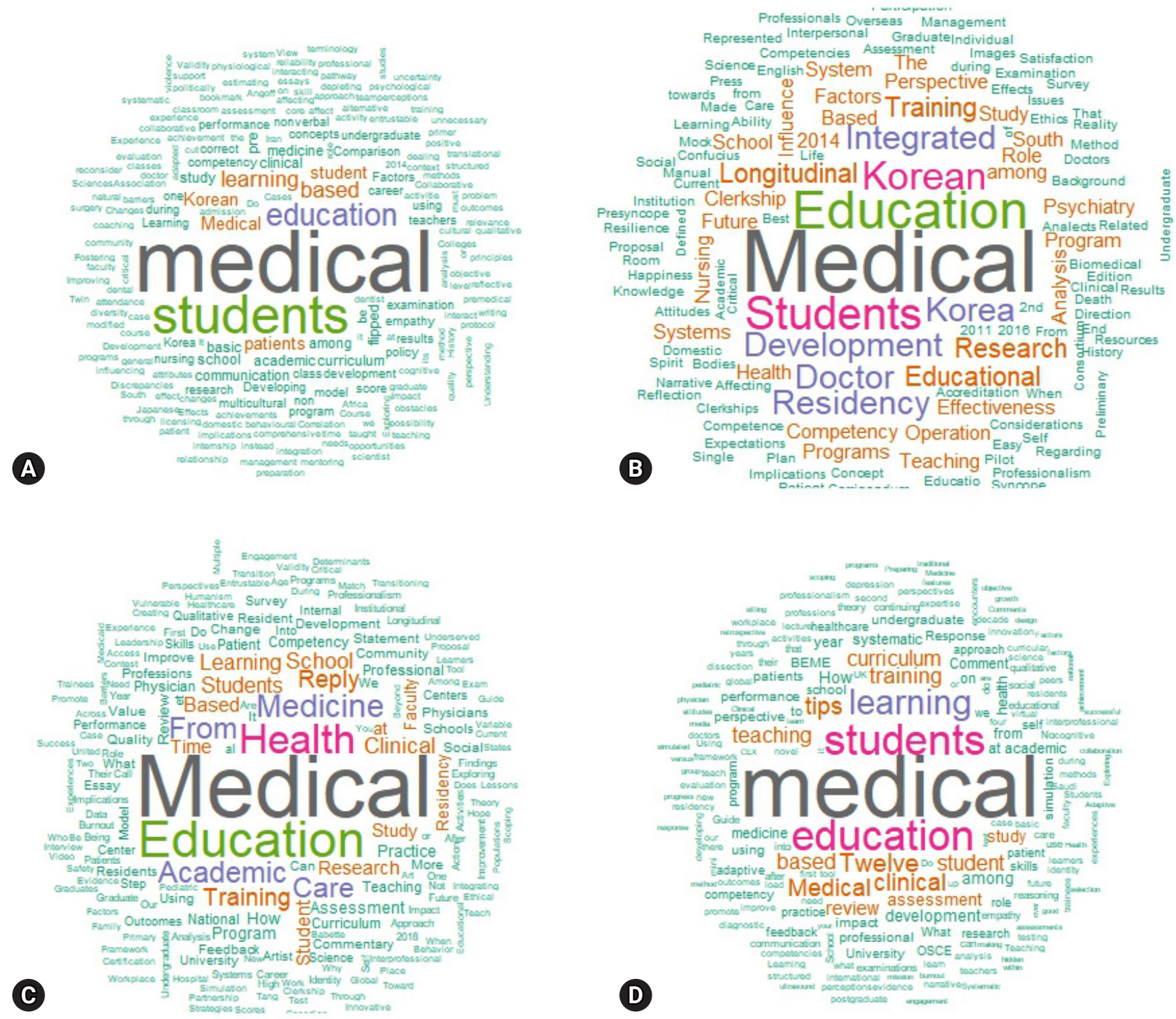

Fig. 1. Word cloud analysis with Rstudio for word frequencies after removing conductions in titles of papers in the KJME (A), KMER (B), Academic Medicine (C), and Medical Teacher (D) during the year 2018. KJME, Korean Journal of Medical Education; KMER, Korean Medical Education Review.

"evaluation" (1.80\%), "teaching" (3.49\%), and "curriculum" (1.50\%). The frequencies in Medical Teacher were "learning" (11.56\%), "clinical” (10.44\%), "skill” (5.10\%), "program" (5.18\%), "evaluation" (2.91\%), "teaching" (8.68\%), and "curriculum" (4.97\%). These results indicate that Korean journals and Medical Teacher have a relatively high proportion of the terms "clinical" and "learning" (7.80\% to $13.66 \%)$, but Academic Medicine has a relatively low proportion $(6.47 \%$ and $4.41 \%$, respectively). Therefore, each journal has a slightly different direction to its pursuits.

\section{Changes in several research topics over 10 years}

According to Kim et al. [8], problem-based learning (PBL) was introduced to Korean medical schools through a research paper that described the Harvard Medical School's curriculum in 1992. Further, a PBL study group was organized under the KSME in 1995, with 15 medical schools applying PBL in their curriculum by 1999 .

Team-based learning (TBL) is a widely used educational 
Table 2. The word frequencies in the title of papers in the KJME and the KMER during year 2009 to 2018

\begin{tabular}{|c|c|c|c|c|c|}
\hline \multicolumn{3}{|c|}{ KJME } & \multicolumn{3}{|c|}{ KMER } \\
\hline Term & No. & $\%$ & Term & No. & $\%$ \\
\hline Medical & 248 & 60.49 & Medical & 117 & 57.07 \\
\hline Student & 157 & 38.29 & Education & 85 & 41.46 \\
\hline Medical student & 105 & 25.61 & Medical education & 53 & 25.85 \\
\hline Medical education & 56 & 13.66 & Student & 45 & 21.95 \\
\hline Education & 88 & 21.46 & Medical student & 24 & 11.71 \\
\hline Clinical & 56 & 13.66 & Clinical & 16 & 7.80 \\
\hline Curriculum & 14 & 3.41 & Curriculum & 19 & 9.27 \\
\hline Evaluation & 24 & 5.85 & Evaluation & 4 & 1.95 \\
\hline Learning & 56 & 13.66 & Learning & 25 & 12.20 \\
\hline Program & 29 & 7.07 & Program & 20 & 9.76 \\
\hline Skill & 33 & 8.05 & Skill & 5 & 2.44 \\
\hline Teaching & 23 & 5.61 & Teaching & 16 & 7.80 \\
\hline
\end{tabular}

KJME, Korean Journal of Medical Education; KMER, Korean Medical Education Review.

Table 3. The word frequencies in the title of papers in the Academic Medicine and the Medical Teacher during year 2009 to 2018

\begin{tabular}{|c|c|c|c|c|c|}
\hline \multicolumn{3}{|c|}{ Academic Medicine } & \multicolumn{3}{|c|}{ Medical Teacher } \\
\hline Term & No. & $\%$ & Term & No. & $\%$ \\
\hline Medical & 1,405 & 30.49 & Medical & 1,602 & 42.78 \\
\hline Education & 791 & 17.17 & Student & 958 & 25.58 \\
\hline Student & 507 & 11.00 & Education & 811 & 21.66 \\
\hline Medical education & 481 & 10.44 & Medical student & 595 & 15.89 \\
\hline Medical student & 326 & 7.07 & Medical education & 493 & 13.16 \\
\hline Clinical & 298 & 6.47 & Clinical & 391 & 10.44 \\
\hline Curriculum & 69 & 1.50 & Curriculum & 186 & 4.97 \\
\hline Evaluation & 83 & 1.80 & Evaluation & 109 & 2.91 \\
\hline Learning & 203 & 4.41 & Learning & 433 & 11.56 \\
\hline Program & 291 & 6.32 & Program & 194 & 5.18 \\
\hline Skill & 94 & 2.04 & Skill & 191 & 5.10 \\
\hline Teaching & 161 & 3.49 & Teaching & 325 & 8.68 \\
\hline
\end{tabular}

method that has been verified in medical education worldwide. It includes such factors as self-directed learning, teamwork, interpersonal communication, peer learning, and feedback, all of which healthcare professionals require. Significant evidence also exists that this method positively impacts academic achievement (e.g., summative assessments). Students prefer TBL over passive lectures or other types of small-group learning [9].

An educational program should be developed to both determine and outline what students can accomplish by the end of the course, and to note what they must learn to achieve target levels of knowledge and performance. Evaluations must then occur to ensure the program's successful implementation [9].

Medical students are highly aware of this academic burden; therefore, it is necessary to explore students' stressful experiences and adaptive endeavors as they progress in their degree programs and the curricula change [10].

Digital devices - such as computers, smartphones, and tablets-are replacing such items as traditional printed books and paper notebooks. Interactive e-learning lectures are also used to supplement traditional lectures' shortcomings in many educational areas. Smartphones have become essential due to the development of mobile devices and wireless communication technologies. Interest has also recently increased in the 4th Industrial Revolution and artificial intelligence (AI), as reflected in the main theme of the KSME's 2017 annual conference, "Future Medical Education and Artificial Intelligence."

Research on these various topics — such as PBL, TBL, program evaluations, burnout, e-learning, and digital-were investigated over a 10-year period, from 2009 to 2018 (Fig. 2).

The concept of PBL was mentioned in 27 papers from the 


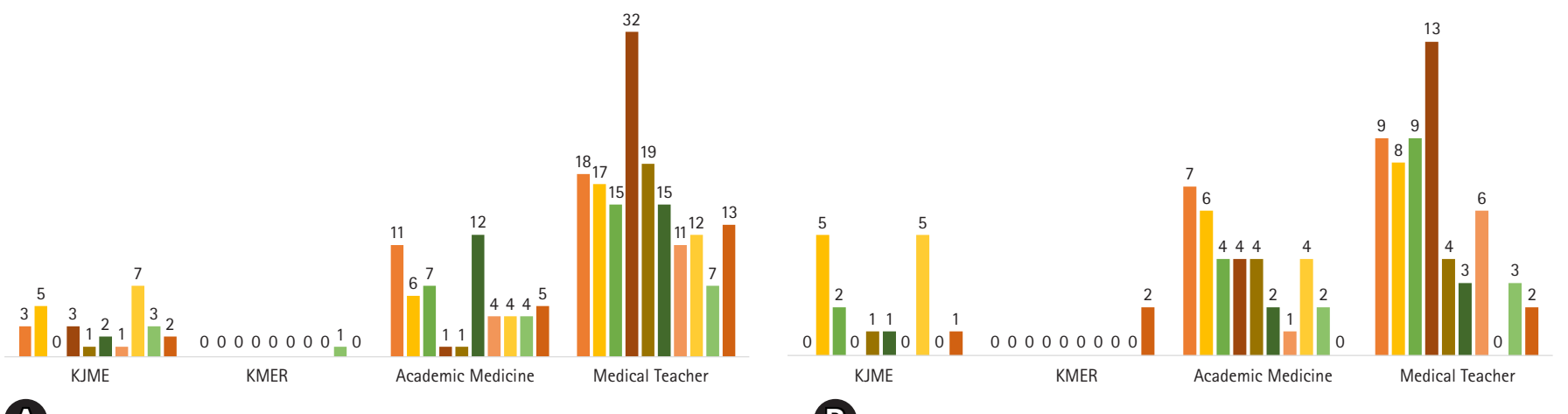

A) $\square 2009 \square 2010 \square 2011 \square 2012 \square 2013 \square 2014 \square 2015 \square 2016 \square 2017 \square 2018$

B $\square 2009 \square 2010 \square 2011 \square 2012 \square 2013 \square 2014 \square 2015 \square 2016 \square 2017 \square 2018$
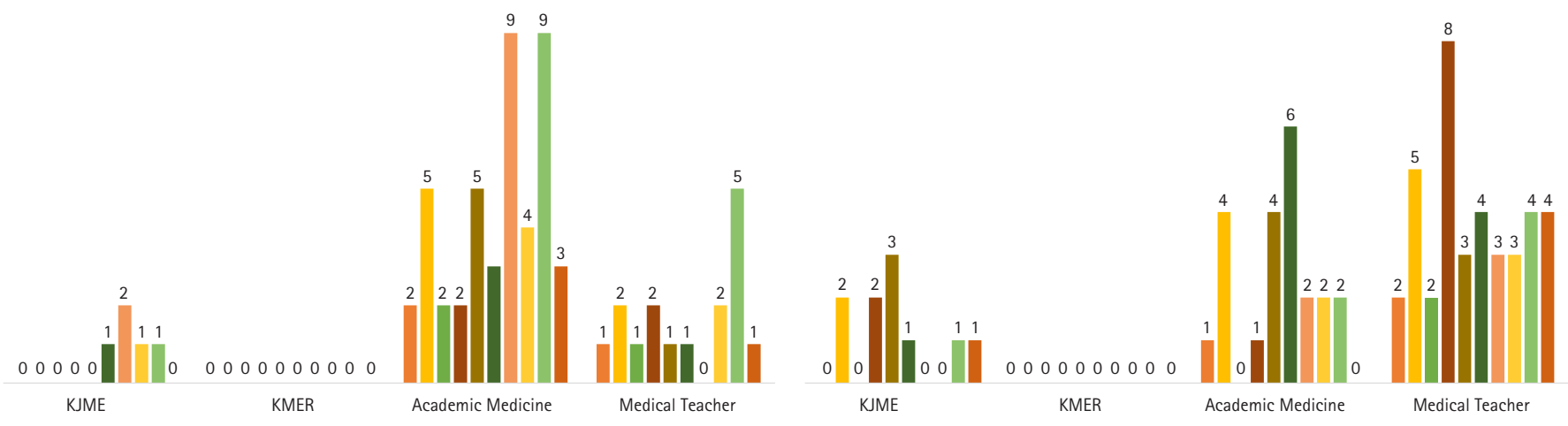

(C)

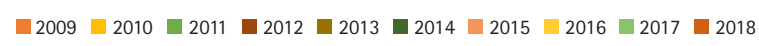

D $\square 2009 \square 2010 \square 2011 \square 2012 \square 2013 \square 2014 \square 2015 \square 2016 \square 2017 \square 2018$

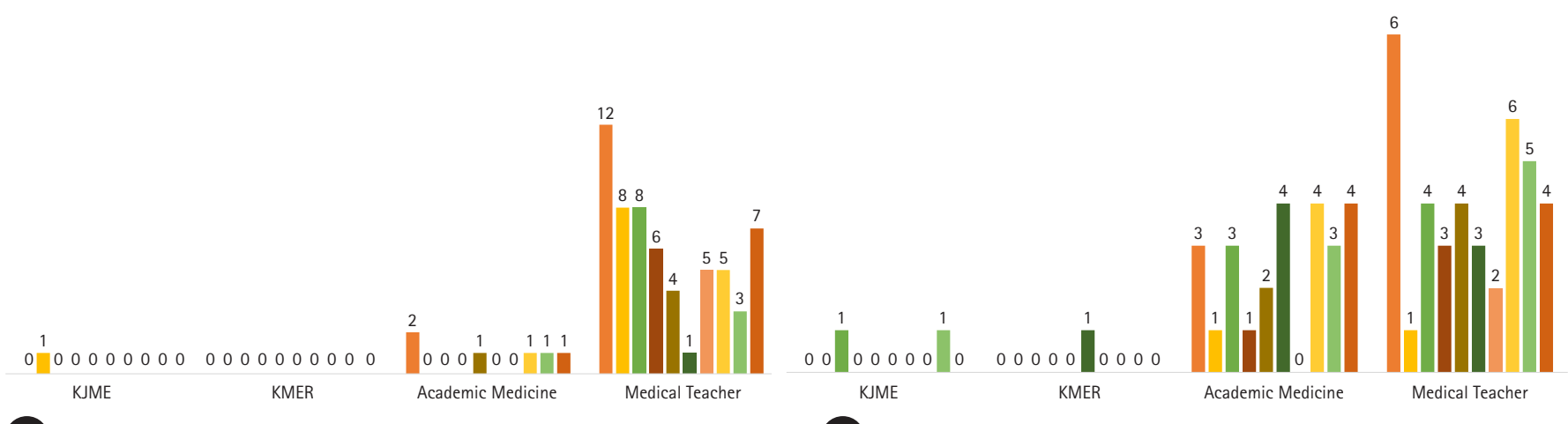

E $\square 2009 \square 2010 \square 2011 \square 2012 \square 2013 \square 2014 \square 2015 \square 2016 \square 2017 \square 2018$

F $\square 2009 \square 2010 \square 2011 \square 2012 \square 2013 \square 2014 \square 2015 \square 2016 \square 2017 \square 2018$

Fig. 2. The number of papers showing MeSH Major Topics, such as "problem-based learning" (A), "program evaluation" (B), and "burnout" (C), and/or such topic words in titles of papers as "team-based learning" (D), "e-learning" (E), and "digital" (F) in the KJME, KMER, Academic Medicine, and Medical Teacher from 2009 to 2018. MeSH, Medical Subject Headings; KJME, Korean Journal of Medical Education; KMER, Korean Medical Education Review.

KJME, 1 from the KMER, 55 from Academic Medicine, and 159 from Medical Teacher. The KJME, Academic Medicine, and Medical Teacher covered PBL almost annually, but the KMER did not. Medical Teacher covered PBL more than Academic Medicine, although the former published fewer papers than the latter. In 2012, Medical Teacher covered PBL in 32 papers, but Academic Medicine discussed PBL in only 1 paper.

While TBL was mentioned in 10 papers from the KJME, 22 from Academic Medicine, and 38 from Medical Teacher, the KMER did not cover TBL. Medical Teacher also covered TBL 
more than Academic Medicine. In 2005, Academic Medicine first covered TBL, and Medical Teacher first covered PBL in 2006. Medical Teacher then covered PBL through 2 to 8 papers every year.

Program evaluations were mentioned in 15 papers from the KJME, 2 from the KMER, 34 from Academic Medicine, and 57 from Medical Teacher. The KJME, Academic Medicine, and Medical Teacher covered program evaluations nearly annually, but the KMER covered only two papers in 2018. Medical Teacher also covered program evaluations annually, ranging from 2 to 13 papers, although not in 2016.

Burnout was mentioned in 5 papers from the KJME, 44 from Academic Medicine, and 16 from Medical Teacher, while the KMER did not cover burnout. The KJME covered burnout the most recently, over 4 years from 2014 to 2017. Academic Medicine covered burnout more than Medical Teacher and did so annually, ranging from 2 to 9 papers.

e-Learning was mentioned often in Medical Teacher, but Academic Medicine and the KJME covered this topic only 6 and 1 times, respectively, while the KMER did not cover it. Medical Teacher covered 59 papers, with every year including 1 to 12 papers.

Digital was mentioned in 2 papers from the KJME, in 2011 and 2017; 1 from the KMER in 2014; 25 from Academic Medicine, and 40 from Medical Teacher. While Academic Medicine covered the digital topic every year, with 1 to 4 papers published each year-except in 2015- Medical Teacher first covered the digital topic annually, ranging from 1 to 8 papers published.

These results illustrate that Medical Teacher seems to primarily discuss teaching and learning methodologies, and Academic Medicine evenly addresses all areas of medical education. As the KJME and KMER publish $1 / 10$ to $1 / 20$ of the number of papers compared to Academic Medicine and Medical Teacher, it is difficult to study all subjects, such as PBL, TBL, program evaluation, burnout, e-learning, and digital. However, as the KJME published research on burnout in the most recent 4 years, it is expected that research on new subjects, such as AI in medical education, will occur in the near future.

\section{Conclusion}

For the 10-year period from 2009 to 2018, Academic Medicine and Medical Teacher published approximately 10 to 20 times more papers compared to the number of papers published in Korean journals. Frequently used words in these titles include "medical," "student," "education," and "learning," seemingly because medical schools direct medical students' education. The frequency of use for such words as "teaching," "learning," "clinical," "eval- uation," "skill," "curriculum," and "program" were investigated; the results reveal that the Korean journals and Medical Teacher have relatively high frequency of "clinical" and "learning" (7.80\% to $13.66 \%$ ), but Academic Medicine has a relatively low frequency (6.47\% and $4.41 \%$, respectively). Therefore, each journal has a slightly different direction in its pursuits.

Researchers investigated various topics, such as PBL, TBL, program evaluation, burnout, e-learning, and digital, over the 10year period from 2009 to 2018. Medical Teacher primarily addressed teaching and learning methodologies, while Academic Medicine handled all areas of medical education.

As the KJME and KMER have $1 / 10$ to $1 / 20$ of the number of papers in Academic Medicine and Medical Teacher, it is difficult to study all subjects. However, it is anticipated that new subjects—such as AI in medical education — will be researched in the near future.

\section{Conflicts of interest}

No potential conflicts of interest relevant to this article was reported.

\section{ORCID}

Seong Yong Kim, https://orcid.org/0000-0003-3419-2699

\section{References}

1. Korean Society of Medical Education (KSME). Abstract books of annual conference [Internet]. Seoul: KSME; 2009-2018 [cited 2019 April 15]. http://www.ksmed.or.kr

2. Association of Medical Education in Europe (AMEE). Links and resources [Internet]. Dundee: AMEE; 2019 [cited 2019 April 15].https://amee.org/links-and-resources

3. Korean Society of Medical Education. The title of papers in Korean J Med Educ [Internet]. 2009-2018 [cited 2019 April 15]. https://koreamed.org/searchresult?q = (TA:"Korean Journal of Medical Education") AND DP:2009/01/01:2018/12/31

4. Yonsei University College of Medicine. The title of papers in Korean Med Educ Rev [Internet]. 2009-2018 [cited 2019 April 15]. http://kmer.or.kr/html/?searchQueryl = strAbstractE\&searchString $1=$ \&mode $=$ search \&pmode $=$ journalsearch \&search $\mathrm{Vol}=\&$ searchIssue $=\&$ searchPage $=\&$ pubyear $=$ notall $\&$ sy ear $=2009 \&$ eyear $=2018 \&$ sort $=$ year \&pageSize $=209$

5. Association of American Medical Colleges (AAMC). The title of papers in Acad Med [Internet]. 2009-2018 [cited 2019 April 15]. https://www.ncbi.nlm.nih.gov/pubmed?ter- 
$\mathrm{m}=\mathrm{Acad}+$ Med $\% 5 \mathrm{BJ}$ our\%5D+AND+2009-2018\%5Bpdat\%5D\&cmd = detailssearch

6. Association of Medical Education in Europe (AMEE). The title of papers in Med Teach [Internet]. 2009-2018 [cited 2019 April 15]. https://www.ncbi.nlm.nih.gov/pubmed/?ter$\mathrm{m}=\mathrm{Med}+$ Teach $\% 5 \mathrm{BJ}$ our\%5D +AND +2009-2018\%5Bpdat $\% 5 \mathrm{D}$

7. RStudio. RStudio [Internet]. Boston: RStudio; 2019 [cited 2019
April 15].https://www.rstudio.com/products/rstudio/

8. Kim YJ, Kang PS, Lee CK, Park JH. The principle and practice of PBL. Korean J Med Educ 2000; 12:1-14.

9. Huggett KN, Jeffries WB. An introduction to medical teaching. 2nd ed. Dordrecht: Springer; 2014.

10. Chun KH, Park YS, Lee YH, Kim SY. Academic burnout and selection-optimization-compensation strategy in medical students. Korean J Med Educ 2014;26:299-308. 